\title{
Optimizing supercritical antisolvent process parameters to minimize the particle size of paracetamol nanoencapsulated in L-polylactide
}

This article was published in the following Dove Press journal:

International Journal of Nanomedicine

27 May 2011

Number of times this article has been viewed

\author{
Mahshid Kalani \\ Robiah Yunus \\ Norhafizah Abdullah \\ Chemical and Environmental \\ Engineering, Faculty of Engineering, \\ University Putra Malaysia, Selangor \\ Darul Ehsan, Malaysia
}

\begin{abstract}
Background: The aim of this study was to optimize the different process parameters including pressure, temperature, and polymer concentration, to produce fine small spherical particles with a narrow particle size distribution using a supercritical antisolvent method for drug encapsulation. The interaction between different process parameters was also investigated.

Methods and results: The optimized process parameters resulted in production of nanoencapsulated paracetamol in L-polylactide with a mean diameter of approximately $300 \mathrm{~nm}$ at $120 \mathrm{bar}, 30^{\circ} \mathrm{C}$, and a polymer concentration of $16 \mathrm{ppm}$. Thermogravimetric analysis illustrated the thermal characteristics of the nanoparticles. The high electrical charge on the surface of the nanoparticles caused the particles to repel each other, with the high negative zeta potential preventing flocculation.

Conclusion: Our results illustrate the effect of different process parameters on particle size and morphology, and validate results obtained via RSM statistical software. Furthermore, the in vitro drug-release profile is consistent with a Korsmeyer-Peppas kinetic model.
\end{abstract}

Keywords: supercritical, antisolvent, encapsulation, nanoparticles, biodegradable polymer, optimization, drug delivery

\section{Introduction}

The morphology, particle size, and distribution of nanoparticles determine drug delivery and bioavailability using these systems, because these characteristics improve the drug solubility in the aqueous media of the body. The pharmaceutical industry needs to produce smaller nanoencapsulated drugs with a narrow size distribution to improve drug delivery using needle-free injections, dry powder inhalers, and controlled-release devices. Using supercritical carbon dioxide $\left(\mathrm{ScCO}_{2}\right)$ as a medium in nanoencapsulation processes is a practical choice, because most pharmaceutical compounds are thermally labile and thus encapsulated drugs should be free from any organic solvent residues and artifacts. In contrast with conventional techniques, the advantages of $\mathrm{ScCO}_{2}$ techniques are their single-step nature, a mild operating temperature, and a very narrow particle size distribution with controlled morphology. ${ }^{1}$ Supercritical fluid technologies are clean and effective methods for drug encapsulation which enable the production of small particle sizes with a narrow distribution, allowing for greater flexibility of drug administration. Furthermore, increasing bioavailability decreases the drug dosage necessary and enables controlled release over a sustained period of time. ${ }^{2-8}$ Small-sized drugs can accelerate towards target organs and be distributed evenly throughout the body. ${ }^{9-13}$ In this study, paracetamol was encapsulated in a biodegradable L-polylactide polymer at different temperatures, pressures, and polymer concentrations using a supercritical 
antisolvent technique. We investigated the effect of these different process parameters on particle size and morphology. The optimum particle size was determined via RSM statistical software and confirmed by scanning electron microscopy.

\section{Methods and materials}

Poly L-lactic acid (intensive viscosity $2.0 \mathrm{dL} / \mathrm{g}$ ) and highpressure liquid chromatography grade methylene chloride were obtained from Sigma-Aldrich (St Louis, MO). Paracetamol (99.9\%) was obtained from the Faculty of Medicine and Health Science, University Putra Malaysia. High-pressure liquid chromatography grade acetone was obtained from Fisher Scientific (Leicestershire, UK). Purified grade carbon dioxide is used as the supercritical fluid.

\section{Preparation of nanoparticles}

Supercritical $\mathrm{CO}_{2}$ was pumped into a high-pressure vessel (490 mL) at specific gas density. The solute was sprayed by a high-pressure pump (SCF P-50A; Thar, Pittsburgh, PA) through a $2 \mu \mathrm{m}$ diameter nozzle. The solvent diffused rapidly from the solution droplets into the bulk supercritical fluid, precipitating the solute. Analysis of variance was performed using RSM software (v6; Maitland, FL). Optimization of particle size was obtained by varying the process conditions. The various process parameters, including pressure, temperature, and polymer concentration used for particle nanoencapsulation are summarized in Table 1 . The operating conditions were then kept constant while varying the other process parameters, as shown in Table 2 .

\section{Characterization}

\section{Scanning electron microscopy}

Scanning electron microscopy (S-3400N; Hitachi, Tokyo, Japan) was used to characterize the morphology and size of the nanoencapsulated particles. Each sample was adhered to adhesive carbon tape on an aluminum stub and coated with gold palladium (Emitech K550X; Quorum Technologies Ltd, West Sussex, UK) at $35 \mathrm{~mA}$ for 2 minutes in a vacuum equal to $1 \times 10^{-4}$ bar.

\section{Thermogravimetric analysis}

Thermogravimetric analysis (model TGA/SDTA851; MettlerToledo, Columbus, $\mathrm{OH}$ ) was used to determine weight loss

Table I Variable process parameters

\begin{tabular}{ll}
\hline Process parameters & Value \\
\hline Pressure (bar) & $80,90,100,110,120$ \\
Temperature $\left({ }^{\circ} \mathrm{C}\right)$ & $30,35,40,45,50,55$ \\
Polymer concentration $(\mathrm{mg} / \mathrm{mL})$ & $12,16,20,24,28$ \\
\hline
\end{tabular}

Table 2 Fixed process parameters

\begin{tabular}{ll}
\hline Process parameters & Value \\
\hline $\mathrm{CO}_{2}$ flow rate $(\mathrm{mL} / \mathrm{min})$ & 15 \\
Feed flow rate $(\mathrm{mL} / \mathrm{min})$ & 1.75 \\
Feed delivery $(\mathrm{mL})$ & 20 \\
Ratio of DCM/acetone & 3.2 \\
Paracetamol concentration $(\mathrm{mg} / \mathrm{mL})$ & 0.5 \\
\hline
\end{tabular}

as a function of incremental temperature. Analysis was performed by increasing the temperature gradually from $50^{\circ} \mathrm{C}$ up to $600^{\circ} \mathrm{C}$ at a rate of $5^{\circ} \mathrm{C} / \mathrm{min}$ and purging the gas with nitrogen at a flow rate of $20 \mathrm{~mL} / \mathrm{min}$.

\section{Zeta potential}

A ZEN 2600 Zetasizer (Malvern Instruments, Worcestershire, UK) with laser diffractometry was used to characterize the zeta potential of the encapsulated paracetamol. A nanoparticle sample weighing $1.5 \mathrm{mg}$ was suspended in $5 \mathrm{~mL}$ of distilled water. The mean value from 20 successive readings was taken as the zeta potential of the paracetamol-L-polylactide nanoparticles.

\section{In vitro drug release}

The paracetamol released into the phosphate-buffered saline medium was analyzed using ultraviolet-visible spectrometry (Model 1650-PC; Shimadzu, Kyoto, Japan) at $243 \mathrm{~nm}$. Nanoencapsulated paracetamol $25 \mathrm{mg}$ was placed into $50 \mathrm{~mL}$ of phosphate-buffered saline ( $\mathrm{pH}$ 7.4) containing sodium azide $0.025 \%$. The sample was placed in a shaking incubator maintained at $50 \mathrm{rpm}$ and $37^{\circ} \mathrm{C}$. Aliquots of $2 \mathrm{~mL}$ were removed from the $50 \mathrm{~mL}$ suspension on various days up to 30 days, and the volume lost was immediately replaced with fresh phosphate-buffered saline.

\section{Results and discussion}

The significant effects constitute the model. The $F$-value in the model was shown to be significant $(F<0.05)$ for A, B, $\mathrm{C}, \mathrm{B} 2, \mathrm{AC}, \mathrm{AB}$, and $\mathrm{BC}$ (where $\mathrm{A}, \mathrm{B}, \mathrm{C}$ are temperature, pressure, and concentration). The following RSM equation was fitted to the response variable (particle size) and three independent variables, ie, temperature (A), pressure (B), and concentration $(\mathrm{C})$ :

Particle size $=+7420.81097+(22.78675 \times \mathrm{A})-(10$ $1.23872 \times \mathrm{B})-(251.88633 \times \mathrm{Conc})+\left(1.86604 \times \mathrm{A}^{2}\right)+$ $\left(0.87703 \times \mathrm{B}^{2}\right)+\left(8.27705 \times \mathrm{C}^{2}\right)-(1.85212 \times \mathrm{A} \times \mathrm{B})+$ $(5.22151 \times \mathrm{A} \times \mathrm{C})-(1.95945 \times \mathrm{B} \times \mathrm{C})$.

The effect of density on particle size is shown in Figure 1. Enhancing supercritical fluid density decreased the particle size. The effect of temperature on particle size 
A

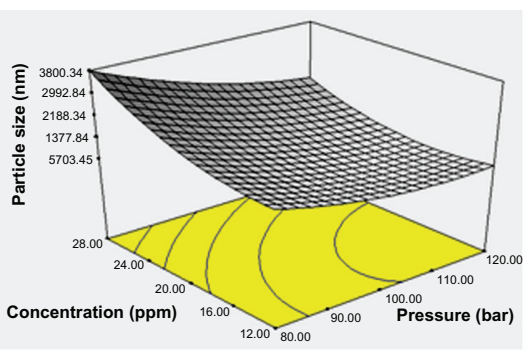

B

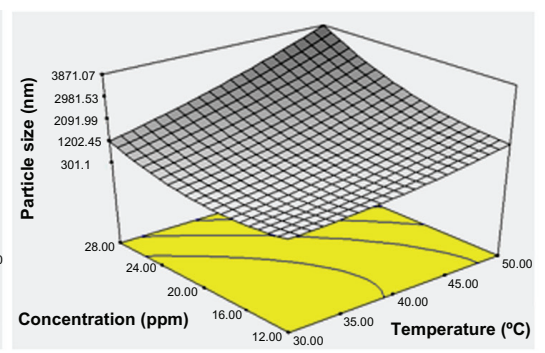

C

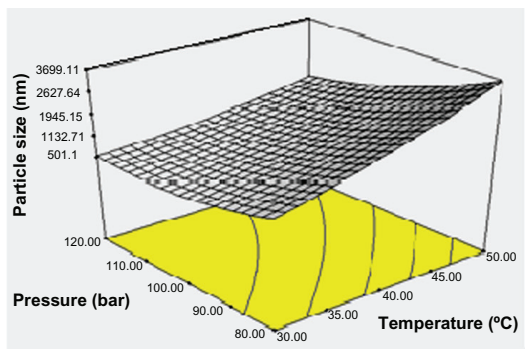

Figure I Response surface plots for particle size as a function of $(\mathbf{A})$ pressure and concentration, $(\mathbf{B})$ temperature and concentration, and $(\mathbf{C})$ temperature and pressure.

was more pronounced at low pressure. The effect of concentration on particle size was different, in that particle size was influenced simultaneously by the degree of super saturation and by the initial concentration. Therefore, it is crucial to adjust the initial polymer concentration. The chemical potential differences between the fluid and solid phases during the supercritical antisolvent process are the driving force for the phase transition, and thus, crystallization. These differences depend on solute activity in the solution at equilibrium. Phase transition occurs spontaneously when there is a negative change in the chemical potential, whereas phase transition is made thermodynamically impossible by a positive change in the free energy. In the thermodynamic equilibrium state, the change in free energy is zero, as follows:

$$
\mu_{3}-\mu_{3}^{*}=R T \ln \left[\frac{\gamma_{3} \chi_{3}}{\gamma_{3}^{*} \chi_{3}^{*}}\right]
$$

where $\mu_{3}^{*}$ is the chemical potential at equilibrium, $R$ is the Boltzman constant, $\gamma_{3}$ is the fugacity (activity) coefficient of the solute at a given concentration $\chi_{3}$, and $\gamma_{3}^{*}$ is the fugacity (activity) coefficient of the solute at equilibrium concentration, $\chi_{3}^{*}$.

The activity coefficient is a function of the pressure and temperature in a supercritical fluid. Based on this equation, it is clear that the presence of $\mathrm{CO}_{2}$ antisolvent dilutes the mixture and decreases the mole fraction of the solute, and thus reduces the chemical potential. Furthermore, the presence of the $\mathrm{CO}_{2}$ antisolvent significantly decreases the equilibrium concentration $\left(\chi_{3}^{*}\right)$. In addition, super saturation in the bulk reactor affects particle growth. The nucleation rate determines both mean particle size and also the total number of particles, and the chemical potential difference is clarified in terms of super saturation, $(S)$, as follows:

$$
\ln S=\frac{\mu_{3}-\mu_{3}^{*}}{R T}=\ln \left[\frac{\gamma_{3} \chi_{3}}{\gamma_{3}^{*} \chi_{3}^{*}}\right]
$$

Therefore,

$$
S=\left[\frac{\gamma_{3} \chi_{3}}{\gamma_{3}^{*} \chi_{3}^{*}}\right]
$$

The super saturation ratio is the solute concentration: solubility ratio or the super saturation concentration. On the other hand, super saturation is a proportion of the degree by which the solute concentration in the solution exceeds the equilibrium concentration at a specified temperature and pressure. If the concentrations of a nonvolatile solute are very low (typically less than $10^{-4}$ mole fraction), the ratio of the fugacity coefficients is approximately equal to 1 and the ratio of supersaturation is equal to the ratio of concentration as follows:

$$
S=\frac{\mathrm{X}_{3}(\chi, T)}{\mathrm{X}_{3}^{*}\left(T, P, \chi_{2}\right)}
$$

where $\mathrm{X}_{3}$ is the mole fraction of solvent.

Based on both statistical data and scanning electron microscopic images, the optimum design point is 120 bar, $30^{\circ} \mathrm{C}$, and a polymer concentration of $16 \mathrm{ppm}$. Scanning electron microscopic images of products produced at temperatures below $45^{\circ} \mathrm{C}$ showed fine spherical particles at all pressures tested. The effect of density on particle size and morphology is shown in Figure 2. The scanning electron microscopic images validate the RSM software results. The thermal stability of nanoencapsulated paracetamol inside the L-polylactide polymer was investigated by thermogravimetric analysis at $120 \mathrm{bar}, 30^{\circ} \mathrm{C}$, and a polymer concentration of $16 \mathrm{ppm}$. From Figure 3, it is evident that the temperature for maximum weight loss of paracetamol from the nanoparticles shifted to $32^{\circ} \mathrm{C}$ due to the L-polylactide polymer nanolayer covering the paracetamol. Furthermore, the thermogram of nanoencapsulated paracetamol inside L-polylactide was similar to that for pure L-polylactide due to the higher ratio of pure polymer in the solute. 
A $35^{\circ} \mathrm{C}, 80$ bar, $16 \mathrm{ppm}$

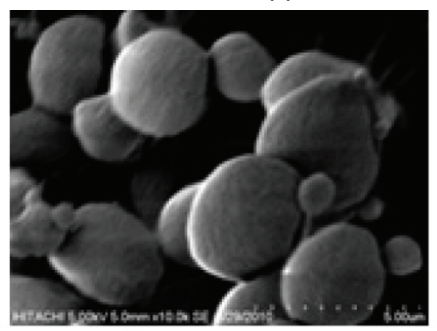

D $35^{\circ} \mathrm{C}, 120$ bar, $12 \mathrm{ppm}$

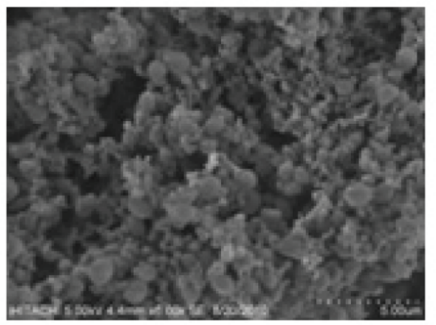

B

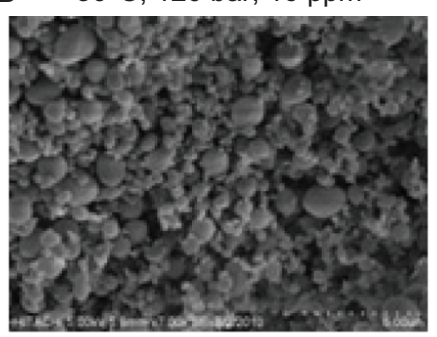

E $\quad 45^{\circ} \mathrm{C}, 100$ bar, $24 \mathrm{ppm}$

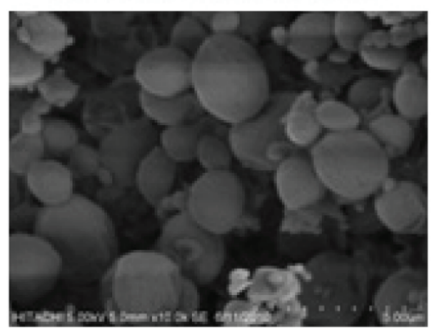

C $50^{\circ} \mathrm{C}, 120 \mathrm{bar}, 16 \mathrm{ppm}$

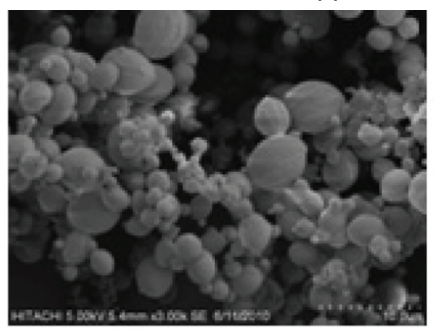

F $\quad 55^{\circ} \mathrm{C}, 100$ bar, $16 \mathrm{ppm}$

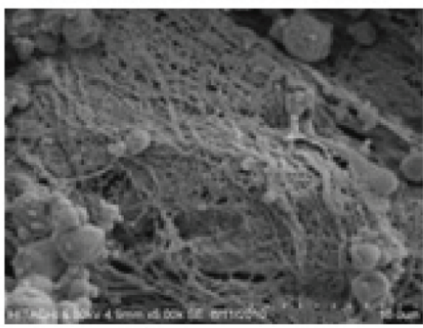

Figure 2 Scanning electron microscopic images of nanoencapsulated paracetamol inside L-polylactide at different process parameters.

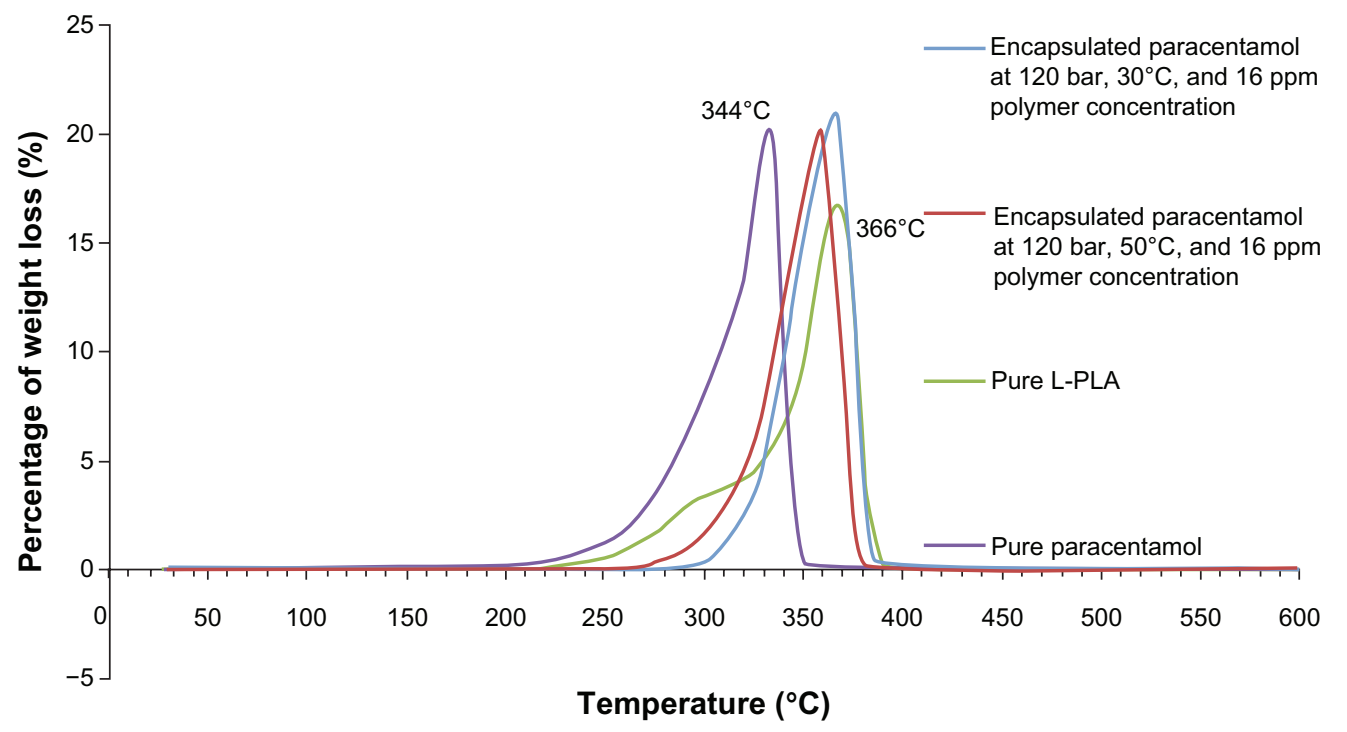

Figure 3 Thermogravimetric analysis of pure paracetamol, pure L-polylactide, and encapsulated paracetamol inside L-polylactide.

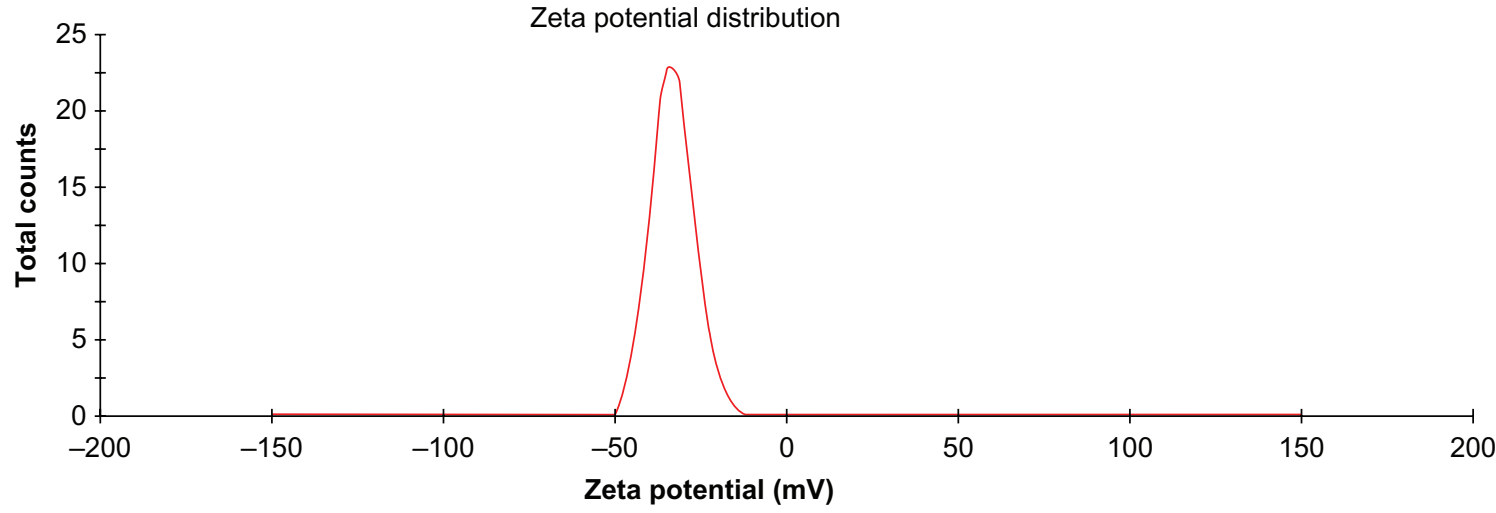

Figure 4 Zeta potential distribution graph of nanoencapsulated paracetamol inside L-polylactide.

Notes: The nanoparticles had a large negative zeta potential $(-33 \pm 3 \mathrm{mV})$ as shown in Figure 4 due to the presence of carboxyl end groups of the L-polylactide chain. Sustained release of paracetamol in phopshate-buffered saline was investigated over 4 weeks. The first burst happened after I week and the cumulative paracetamol release versus time are shown in Figure 5. 


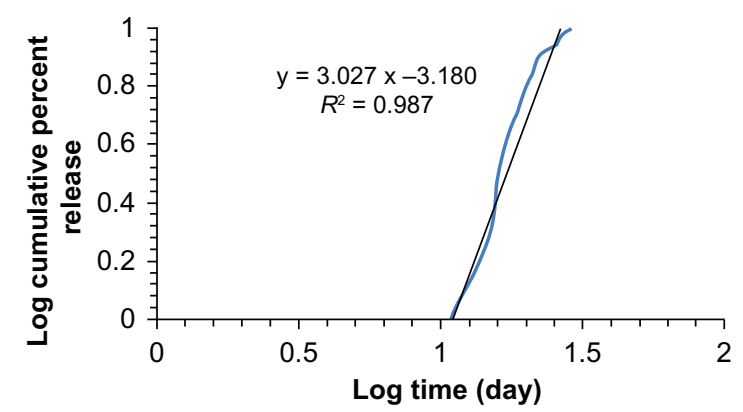

Figure $\mathbf{5}$ In vitro release profile of paracetamol encapsulated in L-polylactide based on the Korsmeyer-Peppas model.

The kinetics were fitted to a Korsmeyer-Peppas model as follows:

$$
\frac{M_{t}}{M_{\infty}}=K \mathrm{t}^{\mathrm{n}}
$$

where $M_{t} / M_{\infty}$ is the fraction of paracetamol release at time $t$, and $k$ is a constant indicating release rate. The slope of the plot indicates the release exponent, $n(n=3.027)$. This shows that the rate of drug release is independent of time and controlled by a swelling mechanism.

\section{Disclosure}

The authors report no conflicts of interest in this work.

\section{References}

1. York P, Kompella UB, Shekunov BY. Supercritical Fluid Technology for Drug Product Development. Swarbrick J, editor. New York:Marcel Dekker, Inc; 2004.

2. Aleksandar MS, Hsu JP, editors. Finely Dispersed Particles: Micro-, Nano-, and Atto-engineering. London, UK: Taylor and Francis; 2006.

3. Kang JW, Wu J, Yin G, et al. Preparation, characterization and in vitro cytotoxicity of indomethacin-loaded PLLA/PLGA microparticles using supercritical $\mathrm{CO}_{2}$ technique. Eur J Pharm Biopharm. 2008;70:85-97.

4. Fages J, Lochard H, Letourneau J, Sauceau M, Rodier E. Particle generation for pharmaceutical applications using supercritical fluid technology. Powder Technology. 2004;141: 219-226.

5. Wu K, Li J. Precipitation of a biodegradable polymer using compressed carbon dioxide as antisolvent. $J$ Supercrit Fluids. 2008;46:211-216.

6. Ginty P, Whitaker M, Shakesheff M, Howdle S. Drug delivery goes supercritical. Nanotoday. 2005; 42-48.

7. Wang Y, Dave RN, Pfeffer R. Polymer coating/encapsulation of nanoparticles using a supercritical anti-solvent process. J Supercrit Fluids. 2004;28:85-99.

8. Thote AJ, Gupta RB. Formation of nanoparticles of a hydrophilic drug using supercritical carbon dioxide and microencapsulation for sustained release. Nanomedicine. 2005;1:85-90.

9. Stephens AP, et al. High-resolution imaging of the supercritical antisolvent process. Exp Fluids. 2005;38:708-719.

10. Patomchaiviwat V, Paeratakul O, Kulvanich P. Formation of inhalable rifampicin-poly(L-lactide) microparticles by supercritical anti-solvent process. AAPS Pharm Sci Tech. 2008;9:1119-1129.
International Journal of Nanomedicine

\section{Publish your work in this journal}

The International Journal of Nanomedicine is an international, peerreviewed journal focusing on the application of nanotechnology in diagnostics, therapeutics, and drug delivery systems throughout the biomedical field. This journal is indexed on PubMed Central, MedLine, CAS, SciSearch ${ }^{\circledR}$, Current Contents ${ }^{\circledR} /$ Clinical Medicine,

\section{Dovepress}

Journal Citation Reports/Science Edition, EMBase, Scopus and the Elsevier Bibliographic databases. The manuscript management system is completely online and includes a very quick and fair peer-review system, which is all easy to use. Visit http://www.dovepress.com/ testimonials.php to read real quotes from published authors. 